\title{
Camalexin Production in Camelina sativa is Independent of Cotyledon Resistance to Sclerotinia sclerotiorum
}

Maria Purnamasari, Gregory R. Cawthray, and Martin J. Barbetti, The University of Western Australia (UWA) Institute of Agriculture and School of Plant Biology, Faculty of Science, UWA, WA, 6009, Australia; and William Erskine and Janine S. Croser, UWA Institute of Agriculture and Centre for Plant Genetics and Breeding, UWA

\begin{abstract}
Purnamasari, M., Cawthray, G. R., Barbetti, M. J., Erskine, W., and Croser, J. S. 2015. Camalexin production in Camelina sativa is independent of cotyledon resistance to Sclerotinia sclerotiorum. Plant Dis. 99:1544-1549.

Camelina sativa (L.) Crantz. has been proposed as a novel source of oilseed resistance to Sclerotinia rot (SR; causal agent Sclerotinia sclerotiorum (Lib.) de Bary). To assess factors likely important in determining the level of resistance to this pathogen, 30 diverse $C$. sativa genotypes were evaluated using a cotyledon test under controlled environmental conditions. Confirmed cotyledon SR-resistant (CS370) and SR-susceptible (CS2305) genotypes were assessed for camalexin production across time following inoculation at the 1-month vegetative stage of growth. There were significant differences among $C$. sativa genotypes in response to inoculation with $S$. sclerotiorum in terms of percent cotyledon disease in-

germplasm and confirmation screening, respectively. Genotype CS370 consistently showed low \%CDI indicating high level of resistance to S. sclerotiorum, whereas CS2305 showed the highest \%CDI value. These findings highlight the potential to develop highly SR-resistant cultivars of $C$. sativa by selection. Furthermore, liquid chromatographic analysis of leaves for both SR-resistant and SR-susceptible genotypes demonstrated that camalexin was produced when inoculated with S. sclerotiorum. However, camalexin production was not linked with disease severity in either genotype, indicating that SR resistance in $C$. sativa is independent of the level of camalexin production.
\end{abstract} dex (\%CDI), with the mean \%CDI ranging from 30.9 to $69.4 \%$ across
Sclerotinia rot (SR), caused by Sclerotinia sclerotiorum (Lib.) de Bary, is one of the most devastating nonspecific diseases of Brassica spp. worldwide (Boland and Hall 1994). This pathogen attacks 408 species, 278 genera, and 75 families of plants, including the economically important oilseed rapeseed (Brassica napus L.) (Barbetti et al. 2014; Bradley et al. 2006). The symptoms of SR mainly appear during flowering and postflowering in rapeseed, because the common route for stem infection is through the infection of the petals (Garg et al. 2010). Stem rot begins as a soft, watery rot on stems (Seguin-Swartz et al. 2009), and affected stems ripen early and can lodge, significantly reducing yield (Rimmer and Buchwaldt 1995). Although rotation with nonhosts and foliar fungicide application can provide limited control, successful management is hampered by the persistent nature of sclerotia in the soil, the broad host range of the fungus, and the high cost of fungicides. Resistance genes are available in the family Brassicaceae (Uloth et al. 2014), and rapeseed cultivars with improved resistance to SR have been developed following introgression of genes from $B$. carinata A. Braun (Uloth et al. 2013) and wild weedy species (Garg et al. 2010). However, the recent characterization of distinct pathogen pathotypes and the fact that host resistance is mostly pathotype dependent has spurred a search for new sources of host resistance that are pathotype independent (Barbetti et al. 2014; Ge et al. 2012). A proposed candidate for pathotype-independent SR resistance is the oilseed Camelina sat$i v a$, a species that has already been introgressed with B. oleracea $\mathrm{L}$. using protoplast fusion to transfer resistance to Alternaria brassicicola (Schwein.) Wiltshire (Sigareva and Earle 1999).

C. sativa is an underutilized crop species receiving renewed attention due to its high omega 3 content in seed and positive agronomic traits (Campbell et al. 2013). The generally low level of general disease incidence in $C$. sativa has been attributed partly to its very

Corresponding author: J. Croser; E-mail: janine.croser@uwa.edu.au

Accepted for publication 22 March 2015.

http://dx.doi.org/10.1094/PDIS-12-14-1297-RE

(C) 2015 The American Phytopathological Society limited area under cultivation and partly from its phytoalexin production (Browne et al. 1991; Putnam et al. 1993). Phytoalexins are chemically diverse antimicrobial compounds that are induced in response to pathogen attack or abiotic stresses. Camalexin (3-thiazol-20-ylindole) is a lipophilic phytoalexin in C. sativa, and is also found in Arabidopsis thaliana (L.) Heynh, A. lyrata (L.), and Capsella bursa-pastoris (L.) Medik (Glawischnig 2007). In addition to this compound, Camelina sativa also synthesizes 6-methoxycamalexin (Browne et al. 1991). First identified in C. sativa, camalexin has been most intensively studied in the model species $A$. thaliana. There are no reports of association between camalexin production in $C$. sativa and SR resistance, and very few studies of genotypic variation of SR resistance in $C$. sativa exist (Seguin-Swartz et al. 2009). A diverse germplasm collection of $C$. sativa assembled in Perth, Western Australia, predominantly sourced from Russia and the Ukraine (Ghamkhar et al. 2010), offers a unique opportunity to identify resistance to important diseases, particularly SR.

Historical studies have demonstrated that S. sclerotiorum inoculation activates jasmonic acid, salicylic acid, and ethylene signaling pathways in Arabidopsis (Guo and Stotz 2007), which are proven to contribute to camalexin production (Denby et al. 2005; Heck et al. 2003; Roetschi et al. 2001, Rowe et al. 2010). Furthermore, Stotz et al. (2011) identified Arabidopsis mutant plants that produced camalexin at low levels and were hypersusceptible to $S$. sclerotiorum infection. This hypersusceptibility may be due to the capacity of $S$. sclerotiorum to detoxify camalexin at lower concentrations via glycosylation to the less toxic derivatives 6-hydroxycamalexin and $6-\beta$-D-glucosylcamalexin (Pedras and Ahiahonu 2002). Camalexin is known to inhibit $S$. sclerotiorum growth at a concentration of $5 \times 10^{-4} \mathrm{M}$ in vitro (Seguin-Swartz et al. 2009), below which camalexin had only a modest effect on viability of this pathogen (Rogers et al. 1996). Thus, it was reasoned that, if plants can produce camalexin at high concentrations, they should, in theory, be resistant to SR.

Therefore, this study was designed to (i) address the paucity of quantitative data regarding the role of camalexin on disease resistance in C. sativa and (ii) determine whether SR resistance exists in $C$. sativa germplasm and whether this species could provide a new source of SR resistance genes for Brassica spp. improvement. To test these objectives, we evaluated the variation across 30 diverse $C$. sativa 
genotypes to $S$. sclerotiorum infection using a cotyledon test. Following the confirmation of cotyledon-resistant and -susceptible genotypes, the relationship between the level of resistance to SR and camalexin production was quantified across time using highperformance liquid chromatography (HPLC) during the vegetative stage of growth.

\section{Materials and Methods}

Experimentation. A cotyledon assay was used to identify the level of pathogen resistance across 30 diverse genotypes of $C$. sativa. The cotyledon assay from Garg et al. (2008) has been shown to be a rapid, reliable, and cost-effective method, and correlates well with field screening results for both rapeseed (Garg et al. 2008) and soybean (Glycine $\max$ (L.) Merrill) (Kim et al. 2000). The results of this germplasm screening enabled the selection of eight genotypes that were rated as putative susceptible (three genotypes) and putative resistant (five genotypes). These eight genotypes were again challenged with the pathogen in a cotyledon assay to confirm the resistance and susceptibility. Then, 1-month-old plants of the most resistant and susceptible genotypes (CS370 and CS2305, respectively) were inoculated with the pathogen and the concentration of camalexin quantified using HPLC in both the diseased and healthy leaf tissue across five time periods $(0,24,48,72$, and $96 \mathrm{~h})$. For each experiment, a completely randomized block design with four replications was adopted and the entire experiment was repeated once.

Genotypes tested. In total, 30 diverse genotypes of $C$. sativa were tested for their camalexin production in the presence or absence of disease caused by $S$. sclerotiorum (Table 1). The accessions were obtained from the N. I. Vavilov Research Institute of Plant Industry, Russia, and originated from five countries: former Czechoslovakia, France, Russia, Sweden, and Ukraine. Accessions were pure-lined, maintained as genotypes in a germplasm collection at the University of Western Australia (UWA), Perth, and are well characterized for agronomic performance, fatty acid composition, and molecular and ecogeographic perspectives (Campbell et al. 2013; Ghamkhar et al. 2010). B. napus 'Mystic' was used as a positive control for S. sclerotiorum pathogenicity and was also obtained from a seed collection held at UWA. Previously, Mystic has been shown to be highly resistant in a cotyledon test (Garg et al. 2008), had small stem lesions in a field trial (Li et al. 2006), and was in the midrange rank across genotypes in another cotyledon test (Uloth et al. 2014).

Plant growth conditions. For germplasm screening, the 30 C. sativa genotypes were grown in a soil mixture composed of finely crushed pine bark/coco peat/sand at 2.5:1.0:1.5 (wt/wt). The potting mix was pasteurized for $90 \mathrm{~min}$. at $65^{\circ} \mathrm{C}$ before use and added to 30cell trays (85-ml Kwikpot Tray, each cell $55 \mathrm{~mm}$ in diameter). Five seeds per genotype were sown in each cell and thinned to two seedlings per cell after emergence. Seedlings were grown until cotyledons were fully expanded, equivalent to growth stage 1.00 on the scale of Sylvester-Bradley and Makepeace (1984), which is around 9 days in C. sativa. Seedling trays were then placed in 35-liter clear plastic storage boxes so that high humidity could be maintained after inoculation, as outlined below. This experimental design was used for confirmation screening in eight genotypes.

For camalexin measurement, the cotyledon-resistant (CS370) and -susceptible (CS2305) genotypes were grown in UWA potting mix in 2-liter pots (140 $\mathrm{mm}$ in diameter) for 4 weeks. Twelve seeds of each genotype were sown in each pot and thinned to 10 seedlings per pot after emergence. All three experiments were conducted within a controlled-environment growth room, set to $18 \pm 1^{\circ} \mathrm{C}$ (day) and $14 \pm 1^{\circ} \mathrm{C}$ (night) on a 12-h light-and-dark cycle, with light intensity of $320 \mu \mathrm{mol}$ quanta $\mathrm{m}^{-2} \mathrm{~s}^{-1}$.

Pathogen. A single $S$. sclerotiorum isolate, MBRS-1, collected from infected tissue of B. napus in the Mount Barker region of Western Australia in 2004 (Li et al. 2006), was used. Isolate MBRS-1 was chosen because it is highly pathogenic (Garg et al. 2008) and belongs to the prevailing pathotype (pathotype 76) occurring in Western Australia (Ge et al. 2012). This isolate has been extensively used for resistance screening of $B$. juncea (L.) Czern and B. napus genotypes in both the glasshouse and the field (Garg et al. 2010; Ge et al. 2012; Li et al. 2006, 2007, 2009).

Inoculum production and inoculation procedure. $S$. sclerotiorum inoculum was prepared as described by Garg et al. (2008). Five agar plug discs of $7 \mathrm{~mm}$ in diameter were cut from the actively growing margin of 3-day-old colonies of $S$. sclerotiorum growing on potato dextrose agar and transferred to a $250-\mathrm{ml}$ flask containing $75 \mathrm{ml}$ of a sterilized liquid medium ( $24 \mathrm{~g}$ of potato dextrose broth, $10 \mathrm{~g}$ of peptone, and 1 liter of $\mathrm{H}_{2} \mathrm{O}$ ). The cultures were rotated at $150 \mathrm{rpm}$ while being maintained at $22^{\circ} \mathrm{C}$. After 3 days, colonies of S. sclerotiorum were harvested and washed twice with sterilized deionized water. The fungal mats were transferred to $125 \mathrm{ml}$ of the same liquid medium and macerated using a handheld blender for $3 \mathrm{~min}$. The macerated mycelial suspension was filtered through four layers of cheesecloth and the concentration adjusted with the same liquid medium to $10^{5}$ fragments $\mathrm{ml}^{-1}$ using a hemocytometer (SUPERIOR) and $0.002 \%$ Tween 20 wetting agent added to ensure effective wetting of the cotyledon surface during inoculation.

A 5- $\mu$ l droplet of fungal suspension at $10^{5}$ fragments $\mathrm{ml}^{-1}$ was deposited on each lobe of each cotyledon of the $30 \mathrm{C}$. sativa genotypes using a micropipette. Deionized water containing $0.002 \%$ Tween 20 wetting agent was deposited on the control plants of all genotypes

Table 1. Response of 30 Camelina sativa genotypes from different global regions to Sclerotinia sclerotiorum expressed as percent cotyledon disease in$\operatorname{dex}(\% \mathrm{CDI})$ at $72 \mathrm{~h}$ postinoculation at the cotyledon stage under controlledenvironment conditions across germplasm and confirmation screening

\begin{tabular}{|c|c|c|c|c|}
\hline Genotype & Species & Country of origin & $\begin{array}{l}\% \text { CDI } \\
\text { Exp. } 1\end{array}$ & $\begin{array}{l}\% \text { CDI } \\
\text { Exp. } 2\end{array}$ \\
\hline $\mathrm{CS} 370$ & $\begin{array}{l}\text { Camelina } \\
\text { sativa }\end{array}$ & Russia & $38.9(1)$ & $32.6(2)$ \\
\hline CS1993 & C. sativa & Russia & $41.7(2)$ & $41.7(5)$ \\
\hline CS4068 & C. sativa & Russia & $42.0(3)$ & $34.0(3)$ \\
\hline CS430 & C. sativa & Russia & $45.5(4)$ & 39.9 (4) \\
\hline CS349 & C. sativa & Russia & $46.2(5)$ & $30.9(1)$ \\
\hline $\mathrm{CS} 253$ & C. sativa & Russia & $47.9(6)$ & $\ldots$ \\
\hline CS4139 & C. sativa & Russia & $48.3(7)$ & $\ldots$ \\
\hline CS4059 & C. sativa & Russia & $48.6(8)$ & $\ldots$ \\
\hline CS4112 & C. sativa & Ukraine & $49.3(9)$ & $\ldots$ \\
\hline CS344 & C. sativa & Russia & $50.4(10)$ & $\ldots$ \\
\hline CS4130 & C. sativa & Russia & $51.0(11)$ & $\ldots$ \\
\hline CS403 & C. sativa & Russia & $51.4(12)$ & $\ldots$ \\
\hline CS4182 & C. sativa & Russia & $51.7(13)$ & $\ldots$ \\
\hline CS4074 & C. sativa & Russia & $51.7(14)$ & $\ldots$ \\
\hline CS3364 & C. sativa & Ukraine & $52.0(15)$ & $\ldots$ \\
\hline CS235 & C. sativa & Russia & $52.0(16)$ & $\ldots$ \\
\hline CS4183 & C. sativa & Russia & $52.8(17)$ & $\ldots$ \\
\hline CS4164 & C. sativa & Sweden & $52.8(18)$ & $\ldots$ \\
\hline CS1330 & C. sativa & Russia & $52.8(19)$ & $\ldots$ \\
\hline CS339 & C. sativa & Russia & $53.1(20)$ & $\ldots$ \\
\hline CS3347 & C. sativa & Ukraine & $53,1(21)$ & $\ldots$ \\
\hline CS2495 & C. sativa & Russia & $53.8(22)$ & $\ldots$ \\
\hline CS4077 & C. sativa & Russia & $53.8(23)$ & $\ldots$ \\
\hline CS1811 & C. sativa & France & $54.2(24)$ & $\ldots$ \\
\hline CS2504 & C. sativa & Russia & $54.5(25)$ & $\ldots$ \\
\hline CS4177 & C. sativa & $\begin{array}{l}\text { Former } \\
\text { Czechoslovakia }\end{array}$ & $54.5(26)$ & $\ldots$ \\
\hline CS4138 & C. sativa & Russia & $56.3(27)$ & $45.1(6)$ \\
\hline CS4111 & C. sativa & Russia & $56.6(28)$ & $\ldots$ \\
\hline CS2292 & C. sativa & Russia & $57.3(29)$ & $52.8(7)$ \\
\hline Mystic & $\begin{array}{c}\text { Brassica } \\
\text { napus }\end{array}$ & Australia & $59.7(30)$ & $66.0(9)$ \\
\hline CS2305 & C. sativa & Russia & $69.4(31)$ & $65.0(8)$ \\
\hline Significance & $\ldots$ & 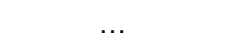 & $P<0.001$ & $P<0.001$ \\
\hline $\begin{array}{l}\text { LSD } \\
\qquad(P<0.05)^{\mathrm{b}}\end{array}$ & $\ldots$ & $\ldots$ & 9.9 & 12.2 \\
\hline
\end{tabular}

${ }^{a}$ Ranking for \%CDI is in parentheses, with 1 for the lowest disease level.

b Least significant difference. 
tested. While inoculating, the mycelial suspension was shaken regularly to maintain a homogenous mixture. Prior to inoculation, cotyledon width was measured with a linear ruler. High humidity $(>95 \%)$ inside the clear plastic storage boxes was achieved by adding a $2.5-\mathrm{cm}$ depth of water at the bottom of the boxes and spraying a very fine mist of water both over cotyledons and on the inside of the container lids. The boxes were sealed within black plastic bags and placed in the controlled-environment growth room, as described by Garg et al. (2008).

Disease assessments. Lesion diameter was measured in millimeters at $72 \mathrm{~h}$ postinoculation (hpi), as described by Garg et al. (2008). In order to accommodate the different cotyledon width diameters among the $C$. sativa genotypes, lesion diameters were converted to a 0 -to-9 scale modified from Williams (1985), where $0=$ no visible symptoms, 1 = necrotic hypersensitive, $2=$ necrotic or water-soaked lesion ( $10 \%$ of total leaf area), $3=$ necrotic or water-soaked lesion (20\%), $4=$ necrotic or water-soaked lesion (20 to 30\%), $5=$ necrotic or water-soaked lesion ( 30 to $40 \%$ ), $6=$ necrotic or water-soaked lesion $(40$ to $50 \%), 7=$ necrotic or water-soaked lesion $(50$ to $60 \%), 8=$ collapsing of cotyledon tissue, and $9=$ collapsing of cotyledon tissue with masses of mycelium. The same scale was also used for scoring the disease level on 4-week-old diseased leaves in the experiments involving camalexin determinations.

The 0-to-9 disease scores were converted into a percent cotyledon disease index (\%CDI) using the method of McKinney (1923), as follows: $\% \mathrm{CDI}=\{[(a \times 0)+(b \times 1)+(c \times 2)+(d \times 3)+(e \times 4)+\ldots(j \times$ $9)] \times 100\} /[a+b+c+d+\ldots j) \times 9]$, where $a, b, c, d, e \ldots j$ are the number of plants with SR disease scores of $0,1,2,3,4, \ldots 9$, respectively. This same method was also used to calculate a percent leaf disease index (\%LDI) in relation to leaf disease.

Camalexin determination. A $t$ test comparison across the two experiments conducted under controlled-environment conditions demonstrated that the relative rankings of genotypes at the cotyledon stage showed a similar overall trend, with CS370 being the most resistant cultivar and CS2305 being the most susceptible. These two genotypes were chosen to determine whether differences occurred in phytoalexin production between resistant and susceptible $C$. sativa genotypes in response to inoculation with $S$. sclerotiorum. For camalexin induction, 4-week-old $C$. sativa plants were sprayed with a suspension of $S$. sclerotiorum at $10^{5}$ fragments $\mathrm{ml}^{-1}$. Until harvest, plants were covered with a transparent plastic hood sprayed with a fine mist of water to maintain high humidity. Because of varied disease symptoms in leaves, even those from the same plant, leaves were categorized and separated into diseased leaves and healthy leaves. The disease severity of the diseased leaves was measured using the \%CDI assessment described above. Camalexin was extracted according to the procedure of Glawischnig et al. (2004). For each measurement, $0.5 \mathrm{~g}$ of fresh leaf tissue was weighed, frozen in liquid nitrogen, and crushed. The extraction was performed twice for $30 \mathrm{~min}$ in $1,000 \mu \mathrm{l}$ of $\mathrm{MeOH}: \mathrm{H}_{2} \mathrm{O}(1: 1)$ at $60^{\circ} \mathrm{C}$. The tissue was removed and the methanol evaporated under vacuum. The aqueous residue was extracted twice with $500 \mu \mathrm{l}$ of chloroform, then combined and dried in a centrifugal evaporator. To the dry residue, $500 \mu$ l of methanol was added;

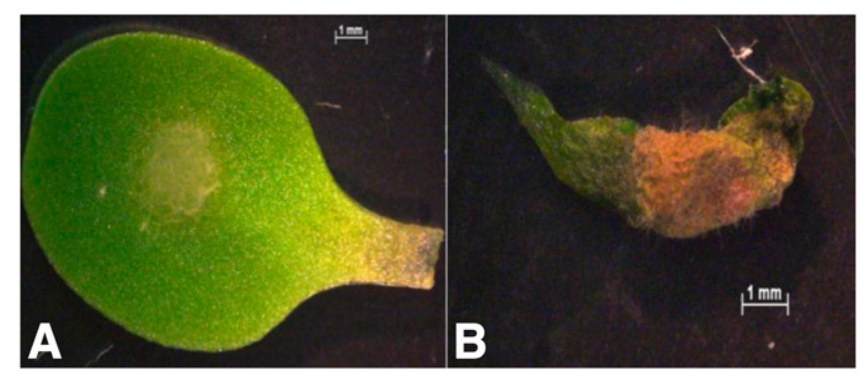

Fig. 1. Cotyledons of Camelina sativa at $72 \mathrm{~h}$ postinoculation ( $\times 20$ magnification). A, White mycelium produced by Sclerotinia sclerotiorum formed around the inoculation point on resistant genotype CS370. B, Abundance of mycelium produced by S. sclerotiorum on leaves of susceptible genotype CS2305. samples were repeatedly vortexed and subsequently used for HPLC analysis.

Sample analysis was performed using HPLC with a 600E pump, 717 plus auto-injector, 996 photodiode array detector, and 470 fluorescence detector (Waters), as adapted from the method of Glawischnig et al. (2004). Separation was achieved at $23 \pm 0.5^{\circ} \mathrm{C}$ on an Alltima C- 18 column $(250$ by $4.6 \mathrm{~mm}$ internal diameter with $5 \mu \mathrm{m}$ packing; Grace

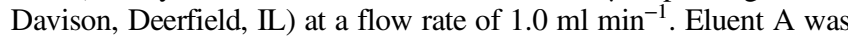
methanol and eluent $\mathrm{B}$ was Milli-Q water, with a gradient elution profile of $60 \%$ A for $1 \mathrm{~min}, 60$ to $100 \% \mathrm{~A}$ in $10 \mathrm{~min}$ (linear), holding at $100 \%$ A for $4 \mathrm{~min}$, followed by an immediate decrease back to $50 \% \mathrm{~A}$ and reequilibration for $15 \mathrm{~min}$. Samples in the auto-injector were held at $10^{\circ} \mathrm{C}$ and the volume injected was $10 \mu l$. Based on authenticated camalexin standards (provided by Prof. Philippe Simoneau [Université d'Angers, Angers, France] and Prof. Erich Glawischnig [Technische Universitat, Munchen, Germany]), the peak at 13.3 min was identified as camalexin and subsequent identification of camalexin in samples was confirmed with the spectral data from the photodiode array detector over the range of 205 to $500 \mathrm{~nm}$. Output from the fluorescence detector $($ excitation $=318 \mathrm{~nm}$ and emission $=370 \mathrm{~nm}$ ) was used for quantification of camalexin, based on a standard curve generated from peak area obtained using synthetic camalexin over a concentration range of 0.1 to $5.0 \mu \mathrm{g} \mathrm{ml}^{-1}$. For each camalexin concentration data point, the assay was performed on four samples and the entire experiment was repeated once.

Statistical analyses. Across experiments, the relationship between the initial and repeat experiment was assessed using a onesided paired $t$ test. If these tests showed no significant differences for these pairings $(P>0.05)$, then the data sets from each experiment were pooled and reanalyzed as a single data set. The \%CDI for cotyledon disease was analyzed using analysis of variance with the statistical program GenStat (14th ed.; VSN International). Fisher's least significant differences $(P<0.05)$ were used to separate significant differences between genotypes. Camalexin determination results were reported as means with their standard errors. Regression analysis was undertaken using the regression function in Microsoft Excel to determine the relationship between camalexin production and \%LDI.

\section{Results}

Variability in cotyledon resistance among diverse germplasm of $C$. sativa. There were significant differences among $C$. sativa genotypes in response to inoculation with $S$. sclerotiorum in terms of $\%$ CDI for germplasm and confirmation screening (Table 1). Small necrotic or soft watery lesions, typical of $S$. sclerotiorum infection, developed on $C$. sativa by $48 \mathrm{hpi}$. In both experiments at $72 \mathrm{hpi}$, the size and severity of lesions varied between genotypes, ranging from small necrotic or water-soaked lesions to collapsed cotyledons with white cottony mycelia on the surface (Fig. 1).

The mean \%CDI on cotyledons across genotypes ranged from 38.9 to $69.4 \%$ in the first experiment and from 30.9 to $64.9 \%$ in the second experiment, with the majority falling into the range of 50 to $57 \%$. When using 30 genotypes, the most resistant genotype was CS370, followed by CS1993 and CS4068. However, CS349 showed the highest level of resistance to S. sclerotiorum in repeated experiments, followed by CS370 and CS4068. In both experiments, CS2305 was the most susceptible. CS2305 was equivalent in terms of its susceptibility to the positivecontrol B. napus Mystic. The \%CDI of both experiments was significantly and positively correlated across repeated entries ( $r=0.89, P<0.001, n=31$; Fig. 2).

Camalexin production of 1-month-old leaves of $C$. sativa in response to $S$. sclerotiorum. For the time course response of resistant and susceptible $C$. sativa genotypes to $S$. sclerotiorum, the HPLC chromatograms of extracts of leaves inoculated with S. sclerotiorum indicated the presence of two compounds that were not present in control leaves. Between these two compounds, compound "I" was produced in detectable amounts at all times in both resistant and susceptible genotypes, whereas the other compound was present in minor quantities and, therefore, was not investigated further. This major compound I with Retention Time $R_{t}=13.3$ min 
was identified as camalexin by comparison with an authenticated synthetic sample.

With CS370 and CS2305, the most resistant and susceptible genotypes among 30 genotypes of $C$. sativa based on the cotyledon test, 1 -month-old leaves produced camalexin when inoculated with $S$. sclerotiorum mycelium at $10^{5}$ fragments $\mathrm{ml}^{-1}$. However, there was no difference in camalexin production between these two genotypes (Table 2; Fig. 3). Furthermore, these leaves developed soft watery lesions approximately $48 \mathrm{hpi}$, which correlated with the time of camalexin induction. Due to differences in disease severity on the leaves, including those from the same individual plant, leaves were separated into two groups: (i) leaves showing disease symptoms and (ii) leaves without disease symptoms, which were considered healthy. The results showed that time, leaf type (healthy versus diseased), and their interaction were all factors influencing camalexin production in this experiment $(P<0.001)$. Both cotyledonary-resistant and -susceptible genotypes produced camalexin in both healthy and diseased leaves. The camalexin levels in healthy leaves were consistently higher than in untreated control leaves, where camalexin induction was below detectable levels ( $<9 \mathrm{ng} / \mathrm{g}$ fresh weight). Moreover, the camalexin production in diseased leaves was higher than in healthy leaves $(P<0.001)$. Induction of camalexin in the resistant genotype (CS370) peaked $(101.4 \pm 21.0 \mathrm{ng} / \mathrm{g}$ fresh weight in diseased leaves) at $48 \mathrm{hpi}$ and then declined significantly thereafter. In contrast, camalexin induction in diseased leaves of CS2305 peaked at 72 hpi with $82.8 \pm$ $28.3 \mathrm{ng} / \mathrm{g}$ fresh weight and then decreased by $96 \mathrm{~h}$. Camalexin concentration decreased as tissue became completely colonized by $S$. sclerotiorum.

In relation to the severity of disease in the diseased leaves of both genotypes, there was no difference in \%LDI between cotyledonresistant and -susceptible genotypes for 1-month-old $C$. sativa plants (Table 2). Furthermore, there was no correlation between camalexin production and \%LDI in both genotypes. The level of camalexin production was independent of \%LDI; for example, two replicates of CS370 had the same \%LDI, 55.6\%, but one replicate produced camalexin at $180.2 \mathrm{ng} / \mathrm{g}$ fresh weight whereas, in the other replicate, the camalexin level was $53.6 \mathrm{ng} / \mathrm{g}$ fresh weight.

\section{Discussion}

Contrary to previous research (Conn et al. 1994; Jejelowo et al. 1991; Sellam et al. 2007; Sigareva and Earle 1999), the lack of relationship between camalexin production and the level of leaf disease suggests that camalexin does not play a major role in SR resistance to C. sativa. However, our hypothesis that $C$. sativa will provide a new source of SR resistance genes for improvement of Brassica spp. was confirmed. For the first time, $C$. sativa has been identified as an excellent source of cotyledon SR resistance genes, which have the potential to either be transferred to Brassica spp. or used for crop improvement within $C$. sativa.

The exact role of camalexin in the expression of resistance of C. sativa to $\mathrm{SR}$ is unclear. Our results demonstrated that camalexin production is independent of the level of SR resistance in $C$. sativa, and our original hypothesis that plants producing camalexin at higher levels will inhibit $S$. sclerotiorum growth and, thus, be resistant to SR was not supported. When camalexin production in resistant CS370 and susceptible CS2305 was compared, the resistant versus susceptible ratings of these two genotypes were not related to the levels of accumulation of camalexin. This finding is similar to those of previous studies. For example, Rouxel et al. (1991) showed there is no correlation between phytoalexin production and disease resistance in a large number of B. nigra (L.), B. rapa (L.), B. juncea, B. carinata, B. oleracea, and B. napus genotypes. Similarly, Pedras and SeguinSwartz (1992) found no clear correlation between phytoalexin accumulation and resistance in rapeseed exposed to virulent or avirulent isolates of the blackleg pathogen Leptosphaeria maculans (Tul.) Ces. \& De Not. Furthermore, the camalexin amounts produced in response to Cochliobolus carbonum R. R. Nelson did not correlate with Arabidopsis resistance (Kagan and Hammerschmidt 2002).

The low level of camalexin production in Camelina sativa and the capability of $S$. sclerotiorum to detoxify camalexin support the argument against a primary role for camalexin in resistance to this pathogen. In the present study, $C$. sativa produced a lower camalexin level than the susceptible line in the study by Sigareva and Earle (1999), which produced camalexin at $1.1 \pm 0.7 \mu \mathrm{g} / \mathrm{g}$ when inoculated with Alternaria brassicicola. Therefore, we propose that phytoalexin accumulation may be part of a coordinated defense strategy, and does not alone account for significant restriction of $S$. sclerotiorum. Furthermore, it is important to note that there is a similar \%LDI between 4-week-old diseased leaves of resistant and susceptible genotypes in

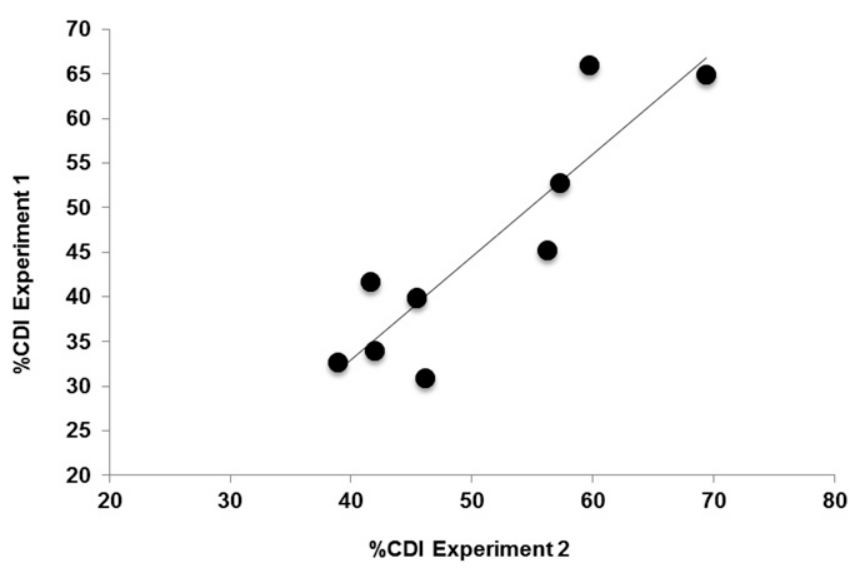

Fig. 2. Correlation between percent cotyledon disease index (\%CDI) of germplasm screening of response of 30 Camelina sativa genotypes to Sclerotinia sclerotiorum and confirmation screening with eight genotypes selected on the basis of putative susceptibility and putative resistance to this fungi.

Table 2. Camalexin production and percent leaf disease index (\%LDI) at 48 , 72 , and $96 \mathrm{~h}$ postinoculation in 4-week-old leaves of Camelina sativa resistant and susceptible genotypes in response to Sclerotinia sclerotiorum

\begin{tabular}{lccc}
\hline Genotype & Time (h) & $\begin{array}{c}\text { Range of camalexin } \\
\text { production (ng/g FW) } \\
\text { in diseased leaves }\end{array}$ & $\begin{array}{c}\text { Range of \%LDI } \\
\text { of diseased leaves }\end{array}$ \\
\hline CS370 & 48 & $40.7-199.9$ & $22.2-66.7$ \\
& 72 & $35.1-114.4$ & $70.4-88.9$ \\
CS2305 & 96 & $19.7-83.7$ & 100.0 \\
& 48 & $31.6-136.1$ & $22.2-50.0$ \\
& 72 & $29.0-272.2$ & $66.7-88.9$ \\
& 96 & $18.7-89.9$ & 100.0 \\
\hline
\end{tabular}

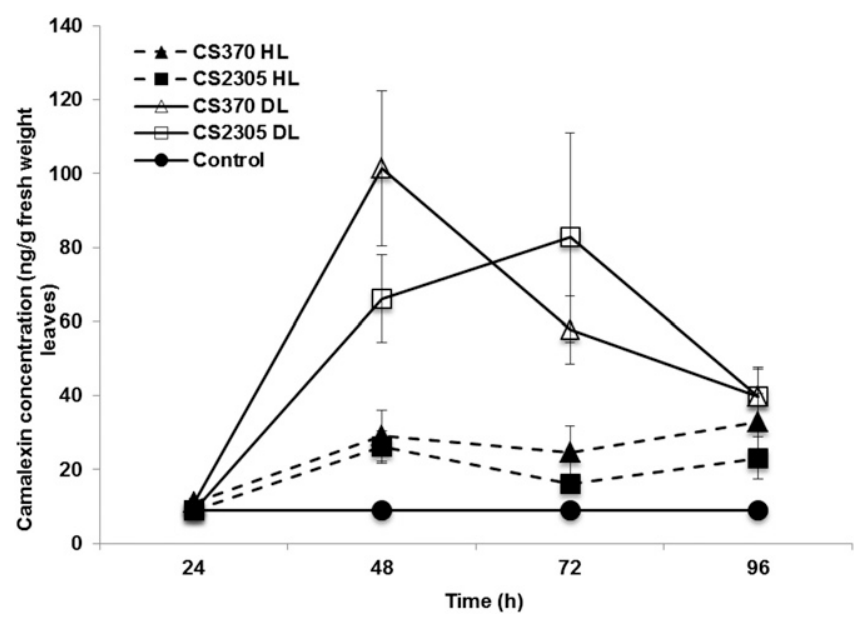

Fig. 3. Camalexin concentration in healthy leaves $(\mathrm{HL})$ of resistant $(\boldsymbol{\Delta})$ and susceptible ( $\square$ ) and diseased leaves (DL) of resistant $(\triangle)$ and susceptible ( $\square$ ) genotypes of 1-month-old plants of Camelina sativa inoculated with Sclerotinia sclerotiorum. Vertical bars represent standard errors of means. 
this study, suggesting that there could be similar camalexin production in both genotypes at this particular growth stage.

Fungal pathogens have a range of different mechanisms to resist and overcome plant defense. For example, S. sclerotiorum can detoxify camalexin via glycosylation (Pedras and Ahiahonu 2002) and $S$. sclerotiorum may produce fungal toxins that determine its pathogenicity against $C$. sativa. For example, Pedras and Ahiahonu (2004) showed that this pathogen produces the phytotoxin sclerin, which causes severe necrosis and chlorosis to B. napus, B. juncea, and Sinapis alba (L.). Sclerotinia sclerotiorum also produces oxalic acid that not only suppresses host oxidative burst and host resistance but also triggers reactive oxygen species-mediated apoptotic-like programed cell death (Hegedus and Rimmer 2005) and plays a detoxifying role in the late infection stages (Heller and Witt-Geiges 2013). Further, Zhu et al. (2013) found that $S$. sclerotiorum produced an integrinlike protein that suppresses the jasmonic/ethylene signal pathwaymediated resistance in Arabidopsis at an early stage of infection. The current study, while not showing a definite relationship between resistance and camalexin production, still accommodates the hypothesis that phytoalexins may play a role, depending upon the inducing agents. For example, Kliebenstein et al. (2005) showed that, whereas camalexin contribution to the Arabidopsis defense response was restricted to camalexin-susceptible isolates of Botrytis cinerea Pers., all isolates of this pathogen could infect a camalexin-deficient mutant. Thus, it would be interesting to do a similar experiment with a pathogen other than $S$. sclerotiorum that is more sensitive to camalexin.

The cotyledon assay successfully distinguished phenotypic variation between $C$. sativa genotypes to SR. Resistant cotyledons had lesions on fewer than $20 \%$ of the cotyledons, on average, and necrotic lesions only developed directly where the inoculum had been applied. Susceptible cotyledons developed large necrotic lesions and usually exhibited full necrosis by $72 \mathrm{hpi}$. A $t$ test comparison between original and repeated experiments showed CS370 to be the most resistant and CS2305 very susceptible. It is noteworthy that the majority of tested $C$. sativa genotypes showed more resistance to S. sclerotinia compared with Brassica napus Mystic. This B. napus genotype has been demonstrated to be moderately to highly resistant to this pathogen in previous cotyledon studies (Garg et al. 2008; Uloth et al. 2014). Therefore, further evaluation of resistance of these C. sativa genotypes to different $S$. sclerotiorum pathotypes is needed in order to locate sources of pathotype-independent host resistance, as has been done for some B. napus breeding populations (Barbetti et al. 2014).

The high correlation coefficient $(r=0.89)$ between germplasm and confirmation screening demonstrates the high degree of reliability of the cotyledon assay when applied to C. sativa. Previously, several methods have been used to quantify against $S$. sclerotiorum in Brassica spp. They include petiole inoculation (Bradley et al. 2006; Zhao et al. 2004), detached leaf inoculation (Bradley et al. 2006; Yu et al. 2010), and an oxalic acid assay (Bradley et al. 2006; Liu et al. 1998). However, resistance identified using these methods is rarely correlated with resistance in field trials. Stem resistance screening methods using a toothpick inoculation or plug inoculation method under greenhouse conditions show high correlation with field resistance (Mei et al. 2012), as does direct field screening using colonized agar plugs attached to stems (Li et al. 2006, 2007, 2009; Uloth et al. 2013). However, both of these techniques require expensive infrastructure or are laborintensive procedures. Thus, the cotyledon assay first developed by Garg et al. (2008), who presented this technique as a reliable means of comparing the level of resistance in B. napus genotypes, and as was later confirmed by Uloth et al. (2014) for a much wider range of crucifer species, has been shown to be an ideal technique for this $C$. sativa study. However, Uloth et al. (2014) revealed that one of the challenges to using this particular assay was in comparing plants with small cotyledon lobes (cotyledon width less than $7 \mathrm{~mm}$ ) such as $C$. sativa with other cruciferous plants with larger cotyledon lobes. Despite differences of cotyledon width among the $C$. sativa genotypes, this was not found to be any impediment to the current study, where cotyledon widths of 6 to $10 \mathrm{~mm}$ needed to be compared with four genotypes with average cotyledon widths $<5$ mm (viz., CS2305, CS3364, CS4111, and CS4112). The current study highlights how this problem can be resolved by converting traditional disease score data into a 0-to- 9 assessment scale modified from that described by Williams (1985). Use of this modified scale enabled successful comparison of 30 genotypes of $C$. sativa and the single B. napus Mystic, despite varying cotyledon lobe sizes.

The lack of correlation between disease indices of the $C$. sativa cotyledons versus 4-week-old leaves supports other evidence that genetic control of resistance to $S$. sclerotiorum is different across plant developmental stages in crucifers. Previously, Zhao and Meng (2003) found that different resistance quantitative trait loci (QTL) play a role in seedling resistance versus stem resistance of rapeseed. This explains why Zhang et al. (2011) found that resistant rapeseed cultivars at the seedling stage could become susceptible at the adult stage and vice versa, demonstrating only QTL qSRS9 at the seedling stage and qSRM9 at the adult stage were at the same position on linkage group N9, whereas other QTL associated with resistance to this fungal pathogen were located in different positions. More recently, studies by Uloth et al. (2014) also suggested the existence of separate genetic control for resistance across diverse crucifers, depending upon plant growth stage. Therefore, although the cotyledon assay in the current study demonstrated genetic variability among $C$. sativa genotypes to SR, the expression of this trait for this species under field conditions remains undetermined.

Despite finding no relationship between camalexin levels and C. sativa resistance level in the current study, camalexin levels in diseased leaves were significantly higher than in healthy leaves. This finding is supported by Bailey (1974) and Smith et al. (1975), suggesting that the accumulation of high concentrations of phytoalexins may be associated with the occurrence of necrotic hypersensitive reaction or larger lesions. The accumulation of camalexin was highly localized. After reaching the maximum concentration peak at $48 \mathrm{hpi}$ in diseased leaves, camalexin production decreased as up to half or more of the leaf tissue became necrotic and colonized by the fungus. Furthermore, it is known that $C$. sativa produces two types of phytoalexins, camalexin and 6-methoxycamalexin (Browne et al. 1991). Although the current study concentrated on camalexin only as the major phytoalexin produced by $C$. sativa, a second compound with spectral properties similar to those of camalexin (based on the spectral data from the photodiode array scan) was also detected. However, due to the lack of standard material for 6-methoxycamalexin, positive identification of this second compound could not be made. Previously, Conn et al. (1994) detected higher production of camalexin and 6-methoxycamalexin in $C$. sativa roots as a response to inoculation with the root rot fungus Rhizoctonia solani Kühn. Thus, it will be interesting to define the role of the latter compound in response to S. sclerotiorum.

This research demonstrating resistance to SR within the germplasm of $C$. sativa adds to the growing body of evidence that $C$. sativa has excellent potential both as a crop in its own right and also as a novel source of disease resistances. The current study confirms the cotyledon assay as a cost-effective and appropriate tool to differentiate resistance against $S$. sclerotiorum. However, the findings of this study do not support the hypothesis that camalexin production would be correlated with the degree of expression SR resistance. Indeed, camalexin alone does not seem to be a major primary factor in limiting the growth of this pathogen in $C$. sativa, and the exact role camalexin plays in the expression of resistance of $C$. sativa warrants further studies.

\section{Acknowledgments}

This work was supported with the funding of the Centre for Plant Genetics and Breeding at the University of Western Australia. Financial assistance to M. Purnamasari was provided through an Australian Awards Scholarship. We thank P. Simoneau, Université d' Angers, France and E. Glawischnig, Technische Universitat, Munchen, Germany for providing us with camalexin standard; The University of Western Australia for providing the facilities; M. P. You for assistance with statistical analyses; and C. Francis for establishing the $C$. sativa germplasm in collaboration with the N. I. Vavilov Research Institute of Plant Industry, Russia. 


\section{Literature Cited}

Bailey, J. A. 1974. The relationship between symptom expression and phytoalexin concentration in hypocotyls of Phaseolus vulgaris infected with Colletotrichum lindemuthianum. Physiol. Plant Pathol. 4:477-488.

Barbetti, M. J., Banga, S. K., Fu, T. D., Li, Y. C., Singh, D., Liu, S. Y., Ge, X. T., and Banga, S. S. 2014. Comparative genotype reactions to Sclerotinia sclerotiorum within breeding populations of Brassica napus and Brassica juncea from India and China. Euphytica 197:47-59.

Boland, G. J., and Hall, R. 1994. Index of plant hosts of Sclerotinia sclerotiorum. Can. J. Plant Pathol. 16:93-100.

Bradley, C. A., Henson, R. A., Porter, P. M., LeGare, D. G., del Rio, L. E., and Khot, S. D. 2006. Response of canola cultivars to Sclerotinia sclerotiorum in controlled and field environments. Plant Dis. 90:215-219.

Browne, L. M., Conn, K. L., Ayer, W. A., and Tewari, J. P. 1991. The camalexins: New phytoalexins produced in the leaves of Camelina sativa (Cruciferae). Tetrahedron 47:3909-3914.

Campbell, M. C., Rossi, A. F., and Erskine, W. 2013. Camelina (Camelina sativa (L.) Crantz): Agronomic potential in Mediterranean environments and diversity for biofuel and food uses. Crop Pasture Sci. 64:388-398.

Conn, K. L., Browne, L. M., Tewari, J. P., and Ayer, W. A. 1994. Resistance to Rhizoctonia solani and presence of antimicrobial compounds in Camelina sativa roots. J. Plant Biochem. Biotechnol. 3:125-130.

Denby, K. J., Jason, L. J., Murray, S. L., and Last, R. L. 2005. Ups1, an Arabidopsis thaliana camalexin accumulation mutant defective in multiple defence signalling pathways. Plant J. 41:673-684.

Garg, H., Atri, C., Sandhu, P. S., Kaur, B., Renton, M., Banga, S. K., Singh, H., Singh, C., Barbetti, M. J., and Banga, S. S. 2010. High level of resistance to Sclerotinia sclerotiorum in introgression lines derived from hybridization between wild crucifers and the crop Brassica species B. napus and B. juncea. Field Crops Res. 117:51-58.

Garg, H., Sivasithamparam, K., Banga, S. S., and Barbetti, M. J. 2008. Cotyledon assay as a rapid and reliable method of screening for resistance against Sclerotinia sclerotiorum in Brassica napus genotypes. Australas. Plant Pathol. 37:106-111.

Ge, X., Yupin, L., Wan, Z., You, M. P., Finnegan, P. M., Banga, S. S., Sandhu, P. S., Garg, H., Salisbury, P. A., and Barbetti, M. J. 2012. Delineation of Sclerotinia sclerotiorum pathotypes using differential resistance responses on Brassica napus and Brassica juncea genotypes enables identification of resistance to prevailing pathotypes. Field Crops Res. 127:248-258.

Ghamkhar, K., Croser, J., Aryamanesh, N., Campbell, M., Kon'kova, N., and Francis, C. 2010. Camelina flax (Camelina sativa (L.) Crantz.) as an alternative oilseed: Molecular and ecogeographic analyses. Genome 53:558-567.

Glawischnig, E. 2007. Camalexin. Phytochemistry 68:401-406.

Glawischnig, E., Hansen, B. J., Olsen, C. E., and Halkier, B. A. 2004. Camalexin is synthesized from indole-3-acetaldoxime, a key branching point between primary and secondary metabolism in Arabidopsis. Proc. Natl. Acad. Sci. 101:8245-8250.

Guo, X., and Stotz, H. U. 2007. Defense against Sclerotinia sclerotiorum in Arabidopsis is dependent on jasmonic acid, salicylic acid, and ethylene signaling. Mol. Plant-Microbe Interact. 20:1384-1395.

Heck, S., Grau, T., Buchala, A., Metraux, J. P., and Nawrath, C. 2003. Genetic evidence that expression of NahG modifies defense pathways independent of salicylic acid biosynthesis in the Arabidopsis-Pseudomonas syringae pv. tomato interaction. Plant J. 36:342-352.

Hegedus, D. D., and Rimmer, S. R. 2005. Sclerotinia sclerotiorum: When "to be or not to be" a pathogen? FEMS Microbiol. Lett. 251:177-184.

Heller, A., and Witt-Geiges, T. 2013. Oxalic acid has an additional, detoxifying function in Sclerotinia sclerotiorum pathogenesis. PLoS One 8:e72292.

Jejelowo, O. A., Conn, K. L., and Tewari, J. P. 1991. Relationship between conidial concentration, germling growth, and phytoalexin production by Camelina sativa leaves inoculated with Alternaria brassicae. Mycol. Res. 95:928-934.

Kagan, I. A., and Hammerschmidt, R. 2002. Arabidopsis ecotype variability in camalexin production and reaction to infection by Alternaria brassicicola. J. Chem. Ecol. 28:2121-2140.

Kim, H. S., Hartman, G. L., Manandhar, J. B., Graef, G. L., Steadman, J. R., and Diers, B. W. 2000. Reaction of soybean cultivars to sclerotinia stem rot in field, greenhouse, and laboratory evaluations. Crop Sci. 40:665-669.

Kliebenstein, D. J., Rowe, H. C., and Denby, K. J. 2005. Secondary metabolites influence Arabidopsis/Botrytis interactions: Variation in host production and pathogen sensitivity. Plant J. 44:25-36.

Li, C. X., Li, H., Siddique, A. B., Sivasithamparam, K., Salisbury, P., Banga, S. S., Banga, S., Chattopadhyay, C., Kumar, A., Singh, R., Singh, D., Agnihotri, A., Liu, S. Y., Li, Y. C., Tu, J., Fu, T. D., Wang, Y. F., and Barbetti, M. J. 2007. The importance of the type and time of inoculation and assessment in the determination of resistance in Brassica napus and B. juncea to Sclerotinia sclerotiorum. Aust. J. Agric. Res. 58:1198-1203.

Li, C. X., Li, H., Sivasithamparam, K., Fu, T. D., Li, Y. C., Liu, S. Y., and Barbetti, M. J. 2006. Expression of field resistance under Western Australian conditions to Sclerotinia sclerotiorum in Chinese and Australian Brassica napus and Brassica juncea germplasm and its relation with stem diameter. Aust. J. Agric. Res. 57:1131-1135.
Li, C. X., Liu, S. Y., Sivasithamparam, K., and Barbetti, M. J. 2009. New sources of resistance to sclerotinia stem rot caused by Sclerotinia sclerotiorum in Chinese and Australian Brassica napus and B. juncea germplasm screened under Western Australian conditions. Australas. Plant Pathol. 38:149-152.

Liu, S. Y., Zhou, B. W., Yu, Q., and Zhou, L. C. 1998. Application of oxalic acid for screening resistance oilseed rape to Sclerotinia sclerotiorum and its affecting factors. Acta Phytophylac. Sin. 25:56-60.

McKinney, H. H. 1923. Influence of soil temperature and moisture on infection of wheat seedlings by Helminthosporium sativum. J. Agric. Res. 26:195-217.

Mei, J., Wei, D., Disi, J. O., Ding, Y., Liu, Y., and Qian, W. 2012. Screening resistance against Sclerotinia sclerotiorum in Brassica crops with use of detached stem assay under controlled environment. Eur. J. Plant Pathol. 134:599-604.

Pedras, M. S. C., and Ahiahonu, P. W. K. 2002. Probing the phytopathogenic stem rot fungus with phytoalexins and analogs: Unprecedented glucosylation of camalexin and 6-methoxycamalexin. Bioorg. Med. Chem. 10:3307-3312.

Pedras, M. S. C., and Ahiahonu, P. W. K. 2004. Phytotoxin production and phytoalexin elicitation by the phytopathogenic fungus Sclerotinia sclerotiorum. J. Chem. Ecol. 30:2163-2179.

Pedras, M. S. C., and Seguin-Swartz, G. 1992. The blackleg fungus: Phytotoxins and phytoalexins. Can. J. Plant Pathol. 14:67-75.

Putnam, D. H., Budin, J. T., Field, L. A., and Breene, W. M. 1993. Camelina: A promising low-input oilseed. Pages 314-322 in: In New Crops. J. Janick and J. E. Simon, eds. John Wiley \& Sons, West Sussex, UK.

Rimmer, S. R., and Buchwaldt, L. 1995. Diseases. Pages 111-140 in: Brassica Oilseeds. D. S. Kimber and D. I. McGregor, eds. CABI International, New York.

Roetschi, A., Si-Ammour, A., Belbahri, L., Mauch, F., and Mauch-Mani, B. 2001. Characterization of an Arabidopsis-Phytophthora pathosystem: Resistance requires a functional PAD2 gene and is independent of salicylic acid, ethylene and jasmonic acid signalling. Plant J. 28:293-305.

Rogers, E. E., Glazebrook, J., and Ausubel, F. M. 1996. Mode of action of the Arabidopsis thaliana phytoalexin camalexin and its role in Arabidopsispathogen interactions. Mol. Plant-Microbe Interact. 9:748-757.

Rouxel, T., Kollmann, A., Boulidard, I., and Mithen, R. 1991. Abiotic elicitation of indole phytoalexins and resistance to Leptosphaeria maculans within Brassiceae. Planta 184:271-278.

Rowe, H. C., Walley, J. W., Corwin, J., Chan, E. K., Dehesh, K., and Kliebenstein, D. J. 2010. Deficiencies in jasmonate-mediated plant defense reveal quantitative variation in Botrytis cinerea pathogenesis. PLoS Pathog. 6:e1000861.

Seguin-Swartz, G., Eynck, C., Gugel, R. K., Strelkov, S. E., Olivier, C. Y., Li, J. L., Klein-Gebbinck, H., Borhan, H., Caldwell, C. D., and Falk, K. C. 2009. Diseases of Camelina sativa (false flax). Can. J. Plant Pathol. 31:375-386.

Sellam, A., Lacomi-Vasilescu, B., Hudhomme, P., and Simoneau, P. 2007. In vitro antifungal activity of brassinin, camalexin, and two isothiocyanates against the crucifer pathogen Alternaria brassicicola and Alternaria brassicae. Plant Pathol. 56:296-301.

Sigareva, M. A., and Earle, E. D. 1999. Camalexin production in intertribal somatic hybrids between Camelina sativa and rapid-cycling Brassica oleracea. Theor. Appl. Genet. 98:164-170.

Smith, D. A., VanEtten, H. D., and Bateman, D. F. 1975. Accumulation of phytoalexins in Phaseolus vulgaris hypocotyls following infection by Rhizoctonia solani. Physiol. Plant Pathol. 5:51-64.

Stotz, H. U., Sawada, Y., Shimada, Y., Hirai, M. Y., Sasaki, E., Krischke, M., Brown, P. D., Saito, K., and Kamiya, Y. 2011. Role of camalexin, indole glucosinolates, and side chain modification of glucosinolate-derived isothiocyanates in defense of Arabidopsis against Sclerotinia sclerotiorum. Plant J. 67:81-93.

Sylvester-Bradley, R., and Makepeace, R. J. 1984. A code for stages of development in oilseed rape (Brassica napus L.). Asp. Appl. Biol. 6:399-419.

Uloth, M., You, M. P., Finnegan, P. M., Banga, S. S., Banga, S. K., Yi, H., Salisbury, P., and Barbetti, M. J. 2013. New sources of resistance to Sclerotinia sclerotiorum for crucifer crops. Field Crops Res. 154:40-52.

Uloth, M., You, M. P., Finnegan, P. M., Banga, S. S., Yi, H., and Barbetti, M. J. 2014. Seedling resistance to Sclerotinia sclerotiorum as expressed across diverse cruciferous species. Plant Dis. 98:184-190.

Williams, P. H. 1985. Crucifer Genetics Cooperative (CrGc) Resource Book. University of Wisconsin, Madison.

Yu, B., Liu, P., Hong, D., He, Q., and Yang, G. 2010. Improvement of Sclerotinia resistance of a Polima CMS restorer line of rapeseed via phenotypic selection, marker-assisted background selection and microspore culture. Plant Breed. 129:39-44

Zhang, J., Qi, C., Pu, H., Chen, S., and Chen, F. 2011. Genetic map construction and Sclerotinia resistance QTLs identification in rapeseed (Brassica napus L.). Proc. 13rd Int. Rapeseed Congress 13:673-676.

Zhao, J., and Meng, J. 2003. Genetic analysis of loci associated with partial resistance to Sclerotinia sclerotiorum in rapeseed (Brassica napus L.). Theor. Appl. Genet. 106:759-764.

Zhao, J., Peltier, A. J., Meng, J., Osborn, T. C., and Grau, C. R. 2004. Evaluation of sclerotinia stem rot resistance in oilseed Brassica napus using a petiole inoculation technique under greenhouse conditions. Plant Dis. 88:1033-1039.

Zhu, W., Wei, W., Fu, Y., Cheng, J., Xie, J., Li, G., Yi, X., Kang, Z., Dickman, M. B., and Jiang, D. 2013. A secretory protein of necrotrophic fungus Sclerotinia sclerotiorum that suppresses host resistance. PLoS One 8:e53901. 